\title{
Estudio de viabilidad de sistemas fotovoltaicos como fuentes de energía distribuida en la ciudad de Arica, Chile
}

Gonzalo D. Valdés-González ${ }^{\star *}$, Emilio R. Rodríguez-Ponce ${ }^{2}$, Christian Miranda-Visa², Jorge Lillo-Sotomayor ${ }^{2}$ (1) Departamento de Ingeniería Industrial y de Sistemas, Universidad de Tarapacá, Chile. Av. 18 de Septiembre 2222, Arica - Chile (correo-e: gvaldes@uta.cl).

(2) Universidad de Tarapacá, Chile. Av. 18 de Septiembre 2222. Arica - Chile

(correo-e: emilior.rodriguez.ponce@gmail.com; c.mirandavisa@gmail.com; jlillo.sotomayor@gmail.com).

* Autor a quien debe ser dirigida la correspondencia

Recibido Dic. 30, 2019; Aceptado Feb. 28, 2020; Versión final Abr. 9, 2020, Publicado Jun. 2020

\begin{abstract}
Resumen
Este artículo analiza las perspectivas de viabilidad económica de instalación de sistemas fotovoltaicos conectados a la red en viviendas de la ciudad de Arica en Chile. Dicha instalación permite al residente de la vivienda generar ingresos mediante la venta de excedentes de energía eléctrica y el ahorro de consumo eléctrico, además de disminuir las emisiones de $\mathrm{CO}_{2}$. La metodología propuesta del estudio se basa en la caracterización de la vivienda promedio de la ciudad de Arica y en el posterior dimensionamiento y análisis de rentabilidad del sistema fotovoltaico a instalar. Se consideran los aspectos económicos, técnicos, legales y ambientales vinculados a la instalación de sistemas fotovoltaicos. Los resultados obtenidos indican que la implementación de sistemas fotovoltaicos como fuente de generación de energía distribuida para las viviendas de la ciudad de Arica, es un proyecto viable, ya que este es rentable a lo largo del periodo analizado.
\end{abstract}

Palabras clave: sistemas fotovoltaicos; energía solar; sistemas on-grid; fuentes de Energía distribuida; evaluación

\section{Viability study of photovoltaic systems as distributed sources of energy in the city of Arica, Chile}

\begin{abstract}
This article analyzes the prospects of economic viability of installing photovoltaic systems connected to the network in homes of the city of Arica (Chile). Installation would allow residents to generate an additional income source by selling electricity surpluses while saving on electricity consumption and reducing $\mathrm{CO}_{2}$ emissions. The proposed methodology is based on the characterization of the average house in the city of Arica and on the subsequent dimensioning and profitability analysis of the photovoltaic system to be installed. This includes the economic, technical, legal, and environmental aspects related to the installation of photovoltaic systems. The results show that photovoltaic systems were profitable in the Arica homes included in this study. In conclusion, the implementation of photovoltaic systems as a source of distributed power generation is a viable project in Arica homes.
\end{abstract}

Keywords: photovoltaic systems; solar energy; on-grid systems; distributed energy sources; evaluation 


\section{INTRODUCCIÓN}

En las décadas 80 y 90 se generó un aumento promedio anual de $0,44 \%$ de las concentraciones de $\mathrm{CO}_{2}$. A partir del año 2000 este promedio aumenta a un 0,55\%, llegando a 400,8 partes por millón (ppm) al año 2015, la mayor concentración desde el inicio de la era industrial, de acuerdo a Earth System Research Laboratory (ESRL, 2016). Esto representa graves consecuencias en el medio ambiente debido a la contaminación y el acelerado calentamiento global (Edenhofer et al, 2012). La contaminación respecto al consumo eléctrico proviene principalmente de la generación centralizada a partir de la quema de carbón, donde no solo se libera $\mathrm{CO}_{2}$ y otros contaminantes (REPNC, 2016), sino que también, debido a las ineficiencias en la producción, junto con las pérdidas de línea de transmisión, los usuarios finales sólo reciben $30 \%-50 \%$ de la energía almacenada originalmente en el carbón (Solar Energy International, 2013).

El desarrollo social y económico esta correlacionado con el aumento de demanda energética. Todas las sociedades requieren de servicios energéticos para cubrir las necesidades humanas básicas y para llevar a cabo los procesos productivos. Aproximadamente, desde el año 1850 la utilización de combustibles de origen fósil (carbón, petróleo y gas) en todo el mundo se ha incrementado hasta convertirse en el suministro de energía predominante, provocando un acelerado aumento de las emisiones del dióxido de carbono $\left(\mathrm{CO}_{2}\right)$.

En Chile son cuatro los sistemas encargados de la transmisión eléctrica: Sistema Interconectado Central (SIC), Sistema Interconectado del Norte Grande (SING), Sistema Eléctrico de Aysén (SEA) y Sistema Eléctrico de Magallanes (SEM). En el caso de la ciudad de Arica, esta pertenece a los precios regulados por el SING. Chile, además de lidiar con problemas medioambientales producto de las emisiones de $\mathrm{CO}_{2}$, las cuales alcanzan un valor sobre 90 mil kilotoneladas el año 2017, ha debido enfrentar problemas de suministro energético durante la última década. Altos y crecientes costos eléctricos debido al alza record del precio de los combustibles fósiles, corte total de suministro de gas natural por parte de Argentina en el año 2008 y calidad de suministros por parte del sistema de distribución. Los efectos de estas problemáticas limitan el crecimiento y desarrollo del país (Corbo y Hurtado, 2014; Pasten, 2012; Pastén y Santamarina, 2012), generando la necesidad en Chile de una política energética: segura, sustentable, eficiente y diversificada. El Ministerio de Energía busca construir una visión compartida para el desarrollo futuro del sector energético con la validación social, política y técnica requerida para transformarse en la política energética que Chile requiere, aumentando hasta en un $70 \%$ la producción total de energía proveniente de Energías Renovables No Convencionales (ERNC) e incrementando los fondos de financiamiento para este tipo de proyectos energéticos (Energía 2050, 2016). Frente a las problemáticas mencionadas, surge entonces como fuente de generación de energía alternativa los sistemas fotovoltaicos (FV), que además de reemplazar el uso de combustibles de origen fósil, generan energía limpia, de calidad y con fuente prácticamente inagotable (Ortega et al, 2010). Existe una amplia literatura de aprovechamiento de la energía solar en distintas aplicaciones, ver por ejemplo Monné et al (2011) y Costa y Ferreira (2007).

Particularmente, la región XV de Arica y Parinacota se considera como una de las zonas geográficas con potencial disponible de energía FV. Posicionándola como un escenario idóneo para el desarrollo y aprovechamiento de este tipo de energía. Dentro de Chile, Arica acapara un $3 \%$ del total del potencial solar del país, correspondiente a 36.647 MW (Santana et al, 2014; Watts et al, 2016; Molina et al, 2017). En cuanto a la generación distribuida, para Arica, entre fines del año 2014 y 2016, se han instalado trece plantas fotovoltaicas adheridas a la ley de generación distribuida, con una potencia en total de 89 kW (CORFO, 2016)

El sistema fotovoltaico a estudiar, se ajusta a la Ley 20.571 de generación distribuida, la cual entró en vigencia el 22 de octubre del 2014, Ley que facilita la venta de excedentes a la red de distribución, y obliga al sistema fotovoltaico cumplir con la norma eléctrica chilena vigente NCh4/2013. La venta de excedentes para la tarifa más común de las viviendas de Arica actúa como Net Billing. Y se destaca que la tarifa vende el excedente a la red eléctrica a un valor $37 \%$ menor al que compra el cliente cada kWh a la empresa distribuidora local. En este escenario, el presente estudio tiene por objeto analizar las perspectivas de viabilidad económica de los sistemas fotovoltaicos conectados a red para el autoconsumo de viviendas de la ciudad de Arica. Se realizará asimismo un análisis de sensibilidad de la rentabilidad de estos sistemas respecto a las principales variables que intervienen.

\section{MATERIALES Y MÉTODOS}

La metodología propuesta para el desarrollo del estudio se basa en la caracterización de la vivienda promedio de la ciudad de Arica y en el posterior dimensionamiento y análisis de rentabilidad del sistema fotovoltaico a instalar, en primera instancia se caracterizara la vivienda promedio en cuanto a su demanda eléctrica mensual, perfil de consumo y plan tarifario. Para seguidamente analizar el recurso solar disponible, a través de la irradiación solar y temperatura. A continuación, se lleva a cabo el dimensionamiento de la instalación fotovoltaica mediante un análisis técnico, el cual incluye la utilización del software PV*SOL® Premium, donde 
se estimará la potencia, producción de energía eléctrica horaria por año y características del diseño del sistema fotovoltaico.

Finalmente se realiza un análisis económico, el cual una vez definidos los parámetros básicos de la inversión, procederá a determinar la rentabilidad de la instalación del sistema fotovoltaico, a través los criterios de evaluación fijados, que contemplan la utilización de los indicadores Valor Actual Neto (VAN), Tasa Interna de Retorno (TIR) (Sapag, 2013) y Costo Nivelado de la Energía (LCOE) (Branker et al, 2011). A su vez se presentara el análisis de sensibilidad realizado sobre los factores que presentan mayor variabilidad, con el objetivo de analizar como se ve afectada la rentabilidad de la inversión para distintos posibles escenarios.

\section{Datos de partida}

Es necesario conocer el perfil de la vivienda promedio en términos del tipo de tarifa, el consumo promedio y el perfil del consumo. Lo anterior con el fin de poder diseñar la instalación fotovoltaica.

\section{Caracterización de la vivienda promedio}

Tarifa eléctrica: La vivienda promedio de la ciudad de Arica está conformada por una cantidad entre 3 o 4 personas (CENSO, 20017) y esta se define como cliente residencial ubicada en el SING. La tarifa eléctrica dominante en la vivienda promedio, es la de Baja Tensión 1 (BT1) con un 98,1\% (de acuerdo a Empresa Eléctrica Arica (EMELARI) en 2015).

Consumo de energía promedio: El consumo eléctrico promedio mensual para una vivienda promedio de la ciudad de Arica es de 180 kilowatt-hora (kWh). De acuerdo a datos de Asociación Gremial de Empresas Eléctricas en 2015, que reúne a las compañías de distribución y transmisión eléctricas a lo largo de Chile. Hoy, está integrada por los grupos Chilectra, Chilquinta, CGE, Saesa y Transelec, además de otras firmas independientes, sumando casi 30 empresas

Perfil de consumo: Para identificar el perfil de la energía consumida por una vivienda promedio de la ciudad de Arica, se escogió una vivienda de características similares a las mencionadas anteriormente. Se procede a utilizar un Egague durante dos semanas del mes de Agosto, instrumento que permite medir y registrar el consumo total de la vivienda en tiempo real. En la Figura 1 se presenta el perfil de consumo promedio, este repite los patrones de las mediciones diarias, existe una constante elevación promedio del consumo entre las $6 \mathrm{am}-8 \mathrm{am}$, con un valor de $0,25 \mathrm{kWh}$ y entre las $6 \mathrm{pm}-8 \mathrm{pm}$, con un valor de $0,3 \mathrm{kWh}$. La mayor parte del consumo es entre las horas de sol ( 7 am y $7 \mathrm{pm}$ ), que corresponde al $59,58 \%$ del total del consumo promedio. Para validar el uso de los datos obtenidos del perfil de consumo promedio presentado anteriormente para todos los meses del año, se procede a analizar la media y varianza de las temperaturas durante las diferentes estaciones del año en la ciudad Arica, ya que este es un factor determinante en el consumo de energía, debido al uso de artefactos eléctricos para calefacción de espacios y/o secadores de ropa (Solar Energy International, 2013). En la Figura 2 se presenta la dispersión presentada por las temperaturas medias de las estaciones del año en la ciudad de Arica, y se observa que las temperaturas medias de cada estación del año se encuentran dentro de los límites de control, por lo que se hace valido el uso del perfil de consumos promedio para todos los meses del año.

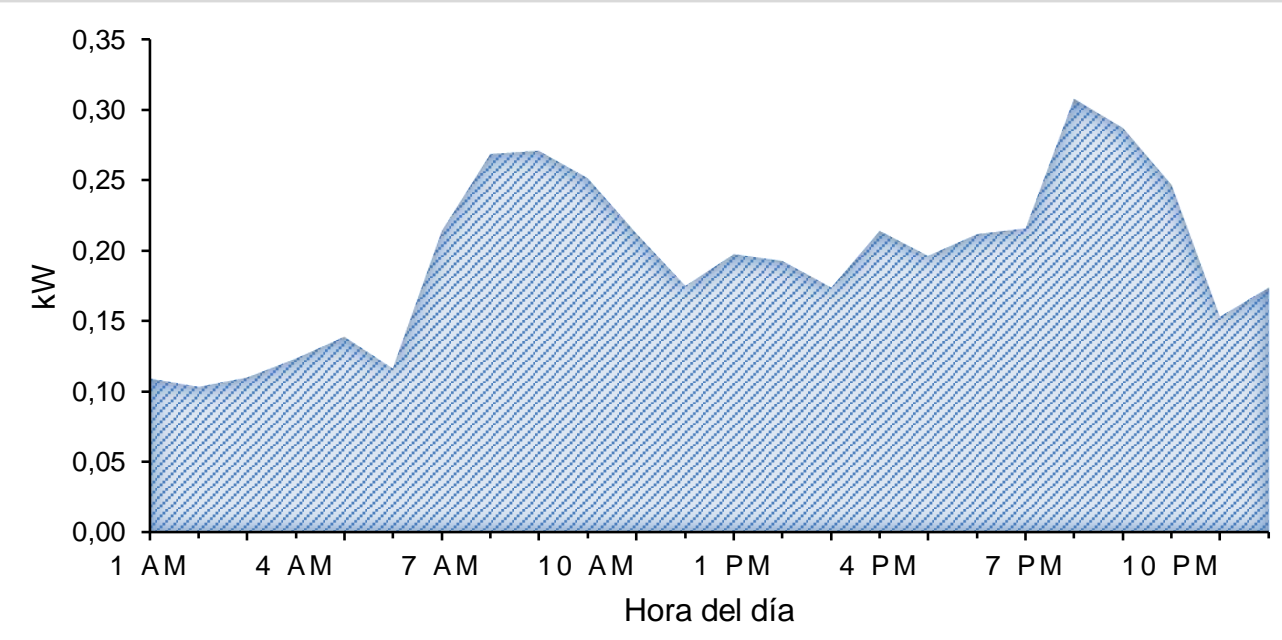

Fig. 1: Perfil de consumo promedio (kW/h) de una vivienda promedio desde el 08-08-016 a 20-08-2016. 


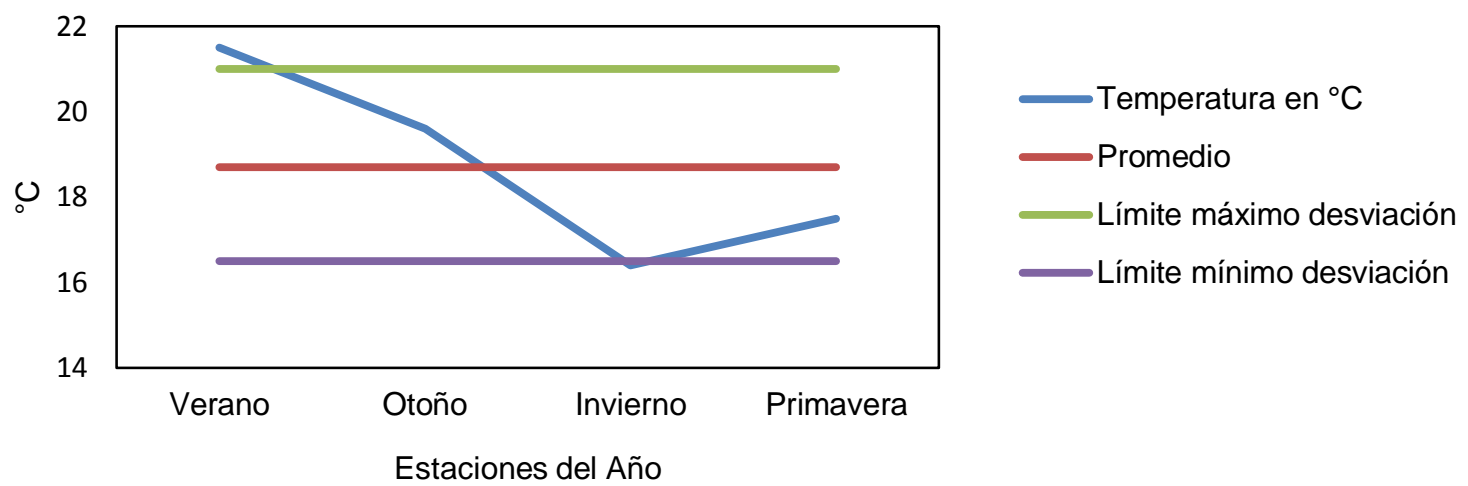

Fig. 2: Variación de temperatura a través de las estaciones del año para la ciudad de Arica.

Recurso solar disponible: Para determinar la producción fotovoltaica es necesario estimar previamente el recurso solar disponible, siendo los principales la radiación e irradiación de la ciudad de Arica. Estos datos son proporcionados por la planta fotovoltaica del Centro de Formación Técnica de Tarapacá (CFT), ubicada en el centro de la ciudad de Arica. La cual se encuentra certificada por la Ley 20.571. Y cuenta con un sistema de monitoreo histórico de la radiación horizontal e Irradiación sobre modulo, entregándonos un valor anual de $2142,7\left(\mathrm{kWh} / \mathrm{m}^{2}\right)$ y $2065,7\left(\mathrm{kWh} / \mathrm{m}^{2}\right)$ respectivamente.

\section{Diseño de la instalación fotovoltaica}

Para determinar el sistema fotovoltaico a instalar se utiliza el software $\mathrm{PV}^{*} \mathrm{SOL} \circledast$ Premium. Programa 3D para el diseño de sistemas fotovoltaicos de origen alemán, el cual recopila datos horarios para producir resultados por hora. Este software presenta alta calidad y precisión en sus resultados (Freeman, y Whitmore, 2014). Incorporando los datos de consumo energético y el recurso solar disponible en la ciudad de Arica al software $\mathrm{PV}^{*} \mathrm{SOL} \circledast$ Premium, este nos entrega los siguientes resultados.

Rendimiento o tamaño del sistema fotovoltaico: Considerando un sistema fotovoltaico conectado a la red con consumidores eléctricos e inyección del excedente en la red y en base a la demanda de la vivienda promedio de $2.160 \mathrm{kWh} / a n ̃ o$. El rendimiento anual específico es de 1.697,08 kWh/kWp. La potencia mínima peak instalada requerida para cubrir la demanda anual es de $1,33 \mathrm{kWp}$, la cual ocupa una superficie de $8,1 \mathrm{~m}^{2}(5$ módulos fotovoltaicos), por lo tanto, la energía anual que generara el sistema mencionado para suplir la demanda es de $2.257 \mathrm{kWh} / a n ̃ o$. Evitando a su vez la emisión de $1.349 \mathrm{~kg} \mathrm{CO} /$ año. Generación y consumo de energía eléctrica del sistema fotovoltaico: $\mathrm{PV}^{\star} \mathrm{SOL} \circledast$ Premium proporciona también como resultado la generación de energía promedio por hora del sistema fotovoltaico y el consumo diario de energía eléctrica de la vivienda promedio para cada uno de los meses a lo largo de un año tipo. La Figura 3 muestra la simulación del sistema para el mes de enero.

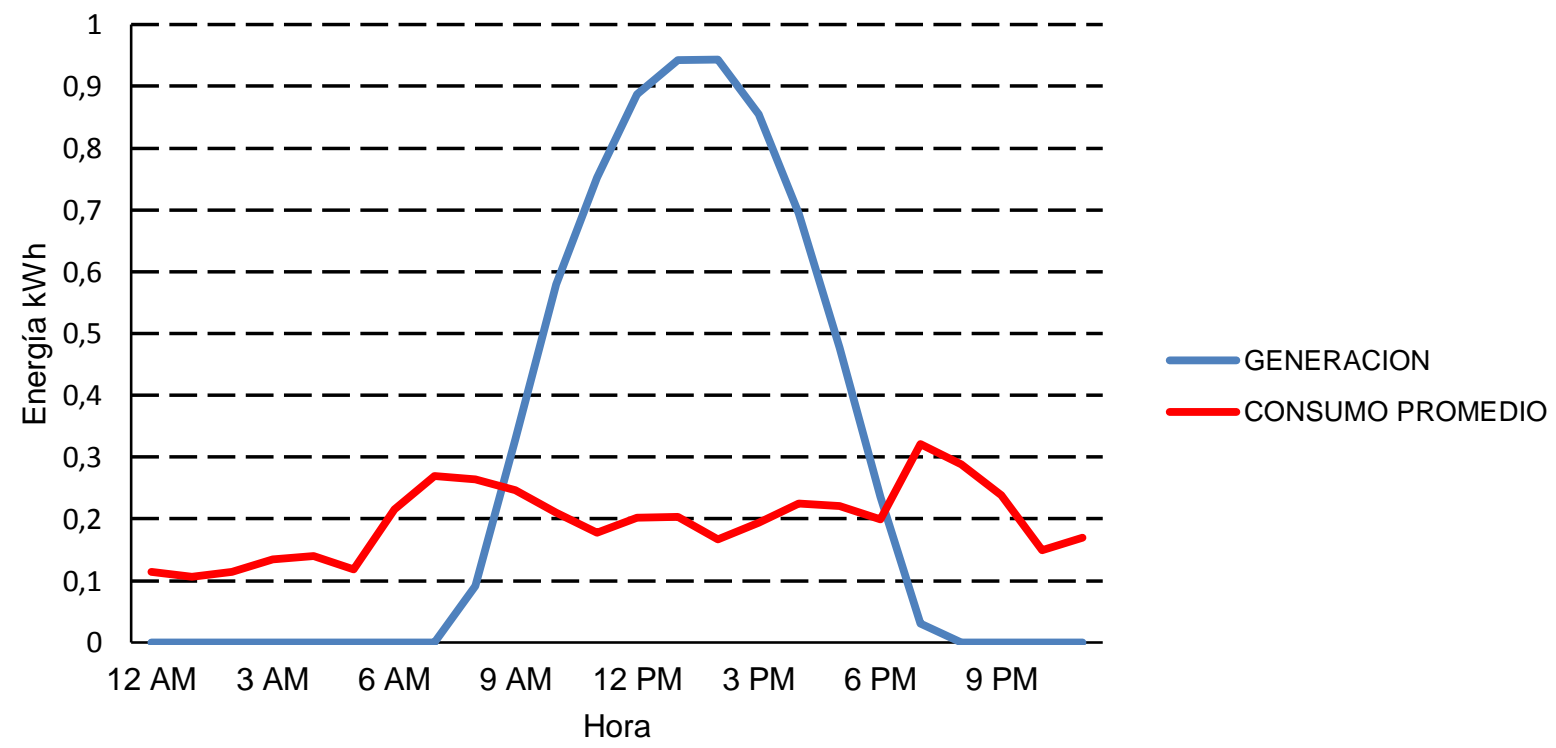

Fig. 3: Mes Enero, Generación vs Consumo promedio. 


\section{Estudio económico y financiero}

El estudio económico realizado basa principalmente en estas cuatro variables: 1) Horizonte de evaluación del proyecto: para este estudio en particular se determinó un periodo de evaluación correspondiente a 25 años, esto debido a que los módulos FV del sistema bajo estudio presentan una garantía de vida y correcto funcionamiento durante dicho periodo de tiempo. Además, este componente es el que representa la mayor parte de la inversión inicial del proyecto; 2) Tasa costo de capital $\left(K_{0}\right)$ : este es determinado mediante la utilización del Modelo de Valoración de Activos (CAPM) y nos estrega un valor de 4,14\%; 3) Tasa de impuesto a la utilidad: se adopta el valor utilizado en el estado de Chile que corresponde al 20\%; y 4) Flujos de caja generados anuales ( $F \$ i)$ : estos flujos corresponden a la diferencia anual de los ingresos y egresos, producidos por el proyecto durante el horizonte de evaluación (Sapag, 2013).

Además se consideran los siguientes antecedentes para la determinación del indicador LCOE: Energía nominal por año: Corresponde a la energía producida del sistema FV, su valor es obtenido del software PV*SOL® Premium y corresponde a 2.257 (kWh/año). Factor de degradación de los módulos FV: Fenómeno natural e inexorable que disminuye la producción de energía. Y de acuerdo a investigación del desempeño de los módulos FV policristalino es de 0,76\% (Skoczek et al, 2009).

\section{Análisis de ingresos y costos}

Análisis de costos: Los costos del proyecto se derivan del tamaño y diseño del sistema FV, estando estos compuestos principalmente por: La inversión inicial, costos operacionales, costos no operacionales y reinversiones. Los cuales se detallan en la Tabla 1. Destacándose la inversión inicial del proyecto, como el mayor costo asociado a la instalación de un sistema FV, con un valor de \$2.731.050.

Tabla 1. Costos asociados al proyecto.

\begin{tabular}{lll}
$\begin{array}{l}\text { Parámetro de } \\
\text { costo }\end{array}$ & $\begin{array}{l}\text { Valor } \\
\text { obtenido }\end{array}$ & Descripción \\
\hline Inversión inicial & $\$ 2.731 .050$ & $\begin{array}{l}\text { Egresos Iniciales requeridos para la puesta en marcha del Proyecto } \\
\text { (Sapag, 2013), en este caso particular se derivan de los siguientes ítems: } \\
\text { Módulos Fotovoltaicos, Inversor, Racking, Medidor, Obras civiles, } \\
\text { Equipamiento, Tramitación SEC. Siendo los módulos FV los de mayor } \\
\text { costo, abarcando un 41\% del total. }\end{array}$
\end{tabular}

\begin{tabular}{|l|l|l|}
\hline $\begin{array}{l}\text { Costos } \\
\text { operacionales }\end{array}$ & $\$ 5.000$ anual & $\begin{array}{l}\text { Estos son los costos que están relacionados con la operación o el } \\
\text { funcionamiento del proyecto (Sapag, 2013), Los cuales normalmente se } \\
\text { dividen en costos variables y costos fijos. Para el caso de un sistema FV, } \\
\text { no se presentan costos que dependan del nivel de producción, es decir se } \\
\text { consideraran únicamente los costos fijos, que corresponden a Limpieza de } \\
\text { paneles cada dos meses. }\end{array}$ \\
\hline
\end{tabular}

Costos no operacionales
\$96.900 anual Estos costos están constituidos por la depreciación, que corresponde, disminución del valor material o funcional de las maquinas, equipos y otros activos fijos que operan en el proyecto a medida que avanza el tiempo (Sapag, 2013). Y es determinado con el "Método de línea recta o lineal", en el cual el valor de los activos se reduce de forma constante y homogénea durante cada periodo de su vida útil.

\begin{tabular}{|l|l|l|}
\hline Reinversiones & $\begin{array}{l}\$ 505.000 \\
\text { cada } 10 \text { años }\end{array}$ & $\begin{array}{l}\text { Reemplazo de equipos debido a la obsolescencia tecnológica según la vida } \\
\text { útil de cada activo fijo (Sapag, 2013). En este caso se debe efectuar una } \\
\text { reinversión del inversor y medidor a usar en el sistema FV. }\end{array}$ \\
\hline
\end{tabular}

Análisis de ingresos: Los ingresos del proyecto se derivan de los ingresos por ahorro de energía eléctrica y de los ingresos por venta de energía eléctrica a la red los cuales, a septiembre del año 2016 tienen un valor de 91,2 y 57, 419 el kWh (valores Empresa Eléctrica de Arica en 2016), estos ingresos varían anualmente y se detallan en la Tabla 2. Se destaca que estos valores van en aumento con el pasar del tiempo llegando a un tope al término del proyecto (año 2041) de $\$ 136.994$ y $\$ 196.083$ respectivamente. 
Tabla 2. Ingresos asociados al proyecto.

\begin{tabular}{l|l|l|}
\hline Parámetro de ingresos & Valor obtenido & Descripción \\
\hline $\begin{array}{l}\text { Ingresos por ahorro de } \\
\text { energía }\end{array}$ & $\begin{array}{l}\text { \$79.80 año 2014 } \\
\$ 136.994 \text { año 2041 }\end{array}$ & $\begin{array}{l}\text { Ahorro monetario producto de la capacidad del sistema FV de } \\
\text { cubrir parte de la energía eléctrica consumida por la vivienda } \\
\text { mediante su generación eléctrica durante las horas de sol. }\end{array}$ \\
\begin{tabular}{|l|l|l|l|} 
Ingresos por venta de \\
energía
\end{tabular} & $\begin{array}{l}\$ 100.291 \text { año 2014 } \\
\$ 196.083 \text { año 2041 }\end{array}$ & $\begin{array}{l}\text { Beneficios monetarios generados mediante la venta de los } \\
\text { excedentes eléctricos que no son aprovechados en el } \\
\text { autoconsumo. }\end{array}$ \\
\hline
\end{tabular}

\section{Criterios de evaluación}

Los criterios a utilizar son tres y corresponden a: 1) Valor Actual Neto (VAN); 2) Tasa Interna de Retorno (TIR); y 3) Costo Nivelado de la Energía (LCOE). El Valor Actual Neto (VAN), parámetro definido como la suma algebraica de los flujos de efectivos $\left(F \$_{i}\right)$ actualizados a la tasa de costo de capital $\left(K_{0}\right)$, considerando tanto los ingresos como los egresos, durante el horizonte de evaluación (i) (Sapag, 2013). Se define según la ecuación (1). Se considera que el proyecto es rentable en términos económicos si el VAN obtenido es superior o igual a cero. Ya que los egresos actualizados serán menores que los ingresos actualizados;

$$
V A N=\sum_{i=0}^{n} \frac{F \$_{i}}{\left(1+K_{0}\right)^{i}}
$$

La Tasa Interna de Retorno (TIR). Este parámetro representa la tasa de descuento que iguala al valor actual de los ingresos y el valor actual de los egresos $\left(F \$_{i}\right)$, es decir, que provoca que el VAN sea igual a cero (Sapag, 2013). Y se define según la ecuación (2). Se considera que el proyecto es rentable en términos económicos si la TIR obtenida es superior a la rentabilidad exigida a la inversión. Ya que el proyecto da una rentabilidad mayor que la rentabilidad mínima requerida.

$$
0=\sum_{i=0}^{n} \frac{F \$_{i}}{(1+T I R)^{i}}
$$

El Costo Nivelado de la Energía (LCOE), parámetro que representa el costo de un sistema de generación de energía durante su vida útil; Se calcula como el precio por kWh en el que la energía debe generarse a partir de una fuente específica durante su vida útil y permite comparar el costo de la generación de energía de diferentes tecnologías (Branker et al, 2011; Watts et al, 2016). Para este estudio se compara el valor obtenido del LCOE y el costo de la energía convencional utilizado por la vivienda. EI LCOE es definido según la ecuación (3), donde $C_{t}$ corresponde a los costos netos del proyecto, $r$ es la tasa de descuento y $E_{t}$ Es la energía del sistema. Sugiriendo que el proyecto es competitivo o viable si el parámetro LCOE resulta tener un valor menor o igual al del costo de la energía convencional que utiliza la vivienda.

$$
L C O E=\frac{\sum_{t=0}^{T} C_{t} /(1+r)^{t}}{\sum_{t=0}^{T} E_{t} /(1+r)^{t}}
$$

\section{RESULTADOS}

Los resultados obtenidos del flujo de caja del proyecto son los siguientes: VAN $=\$ 44.044$. TIR $=4,28 \%$. Considerando el flujo de caja obtenido, además de la energía nominal generada por año y el Factor de degradación se obtiene: $\mathrm{LCOE}=103,34(\$ / \mathrm{kWh})$. Por lo tanto y de acuerdo con los criterios de evaluación, el proyecto es rentable y se debe aceptar, debido a que el VAN es mayor que cero y además la TIR es mayor que el costo de capital. En cuanto al LCOE este se encuentra por debajo del precio convencional de energía ( $\$ / \mathrm{kWh}$ ) consumida por la vivienda promedio de la ciudad de Arica por lo que es factible y por ende viable instalar un sistema fotovoltaico con las características entregadas en el presente estudio.

Se han seleccionado las siguientes variables para realizar los análisis de sensibilidad: Los ingresos operacionales, el costo de capital $\left(K_{0}\right)$ y la inversión inicial $\left(\mathrm{l}_{0}\right)$. Para los ingresos operacionales se considerarán casos optimista y pesimista respecto de la proyección del precio de compra y venta de energía lo cual considera un aumento o disminución promedio de 19\% respectivamente de los ingresos operacionales. 
Para el costo del capital se consideran dos casos en los cuales el costo aumenta un $2 \%$ y un $4 \%$. En el caso de la inversión inicial se consideran dos casos donde se incrementa la inversión inicial en $11 \%$ y $22 \%$.

Para cada una de ellas se procede a obtener el VAN, TIR Y LCOE para distintos valores de dicha variable, dentro de un rango de variación razonable, comparando en primera instancia los ingresos operacionales vs costo de capital y posteriormente los ingresos operacionales vs inversión inicial del sistema FV bajo estudio, entregándonos los diferentes posibles escenarios. Los valores obtenidos para el VAN y TIR presentan ambos una situación favorable solo para los casos de ingreso operacional normal y optimista con un costo de capital del $4,14 \%$, para el resto de casos observados solo se obtienen valores negativos y desfavorables para el proyecto. Los valores obtenidos para el LCOE se aprecia un alza del 13\% si el costo de capital aumenta a un 6,14\%, generando que el LCOE esté por encima del precio al que se enfrentaría el cliente BT1 sin sistema FV durante los primeros cinco años del proyecto. Y un alza del $28 \%$ del LCOE para un costo de capital del $8,14 \%$, generando que el LCOE obtenido, este por encima del precio al que se enfrentaría el cliente BT1 sin sistema FV durante los primeros diez años del proyecto. Ambos casos son menos favorables que la situación inicial propuesta. Nuestros resultados complementan y potencian los resultados propuestos en Ortega et al (2010), Watts et al (2016) y Molina et al (2017).

\section{CONCLUSIONES}

De acuerdo al trabajo presentado y a los resultados obtenidos, se pueden plantear las siguientes conclusiones principales:

El Norte del territorio chileno y específicamente la ciudad de Arica, presentan un gran potencial y características idóneas para la implementación, aprovechamiento y desarrollo de la energía solar FV.

El valor del precio de inyección de energía de la vivienda a la red eléctrica es siempre menor que el valor del precio de compra de energía eléctrica por parte de la vivienda a la red eléctrica, esto a lo largo de todos los meses del año. Por lo cual se hace evidente que es más conveniente concentrar la mayor cantidad de demanda energética en las horas de sol, cuando el sistema FV está en operación, en vez de vender la electricidad generada a la red eléctrica.

Con la ayuda del estudio técnico se definió un sistema FV con una potencia peak de 1,33 kWp que consta de 5 paneles FV de $265 \mathrm{Wp}$, un inversor de $1,5 \mathrm{~kW}$, una estructura o racking y un medidor bidireccional. Dicho sistema presenta una generación de energía eléctrica estable y sin grandes variaciones durante todo un año.

La evaluación económica de implementación de sistemas FV como fuente de generación de energía distribuida para las viviendas de la ciudad de Arica, es un proyecto viable, ya que este es rentable a lo largo del periodo analizado. Y el costo de la energía para este tipo específico de tecnología está por debajo del costo convencional de electricidad consumida por la vivienda promedio de la ciudad de Arica a lo largo de los 25 años.

Según los resultados obtenidos en el análisis de sensibilidad, esta iniciativa de proyecto es altamente sensible a las variables de Costo de Capital y a su vez a los valores futuros del precio de compra y venta de energía eléctrica, los cuales hacen cambiar la condición del proyecto a no viable.

\section{NOTACIÓN}

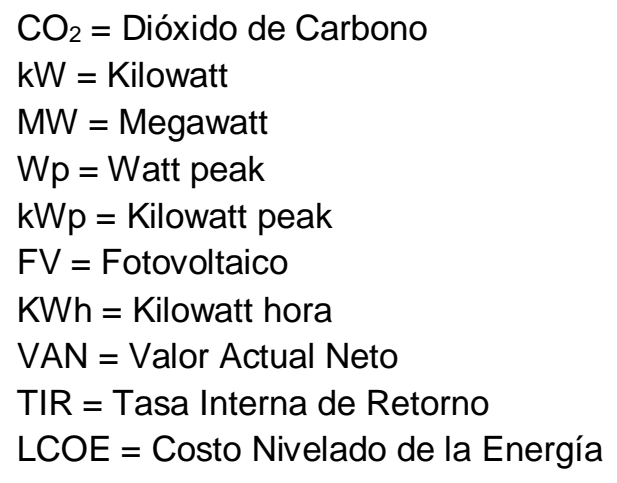

\section{AGRADECIMIENTOS}

El autor de correspondencia agradece el apoyo de los Proyectos de Investigación Científica y Tecnológica para Estudiantes de Pregrado UTA 2019 (códigos 8745-19 y 8746-19). 


\section{REFERENCIAS}

Branker, K., Pathak, M. J. M., Pearce, J. M., A Review of Solar Photovoltaic Levelized Cost of Electricity. Renewable Sustainable Energy Rev., 15, 4470-4482 (2011).

Censo., Instituto Nacional de Estadisticas, (2017).

Corbo, V., Hurtado, A., Causas y consecuencias del problema energético en Chile: Una visión desde la macroeconomía. Puntos de Referencia 382: 1-16 (2014).

CORFO., National Centre for Innovation and Promotion of Sustainable Energy (CIFES), (2016).

Costa, A. R., Ferreira, S. R., Sistema de Secado Solar para Frutos Tropicales. Inf. tecnol.,18(5), 49-58 (2007).

ESRL., Earth System Research Laboratory, Global Monitoring Division. (2016)

Edenhofer, O., Pichs-Madruga, R. y otros seis autores, Renewable Energy Sources and Climate Change Mitigation, doi : 10.1017/CBO9781139151153, Cambridge: Cambridge University Press, (2011).

Energía 2050., Energía 2050 política energética de Chile. Ministerio de Energía (2016).

Molina, A., Falvey, M., Rondanelli, R., A solar radiation database for Chile Sci. Rep., 7. Doi: 14823. 10.1038/s41598-01713761-x, (2017).

Monné, C., Alonso, S., Palacín, F., Evaluación de una Instalación de Refrigeración por Absorción con Energía Solar. Inf. tecnol., 22(3), 39-44 (2011).

Ortega, A., Escobar, R., Colle, S., De Abreu, S. L., The State of Solar Energy Resource Assessment in Chile. Renew. Energy 35, 2514-2524 (2010).

Pastén, C., Chile, energía y desarrollo. Obras y Proyectos, (11), 28-39. https://dx.doi.org/10.4067/S071828132012000100003, (2012).

Pastén, C., Santamarina, J. C., Energy and Quality of Life, doi: 10.1016/j.enpol.2012.06.051, Energy Policy, 49, 468-476, (2012).

REPNC, Renewable Energy Policy Network for the 21st Century, Global Status Report. Comité anual, volumen XI, páginas 16-21, 60- 66 (2016).

Santana, C., Falvey, M., Ibarra, M., y Garcia, M., Energías Renovables en Chile: El potencial Eólico, Solar e Hidroeléctrico de Arica a Chiloé Tech. Rep. 978-956-8066-15-4, Ministerio de Energia, (2014).

Sapag, N., Sapag, R., Preparación y Evaluación de Proyectos. Quinta edición, McGrall-Hill Interamericana, Bogotá, Colombia (2013).

Skoczek, A., Sample T., Dunlop E. D., The Results of Performance Measurements of Field-Aged Crystalline Silicon photovoltaic modules Prog Photovolt: Res Appl, 17, pp. 227-240 (2009).

Solar Energy International, Solar Electric Handbook: Photovoltaic Fundamentals and Applications. Custom edition, Pearson Learning Edition, Boston, United States of America. pp. 7-20 (2013).

Watts D., Oses, N., Pérez, R., Assessment of Wind Energy Potential in Chile: A Project-Based Regional Wind Supply Function Approach, Renewable Energy, Volume 96, Part A, Pages 738-755, ISSN 0960-1481 (2016). 